\title{
PRINCÍPIOS DO SISTEMA ÚNICO DE SAÚDE: compreensão dos enfermeiros da Estratégia de Saúde da Família
}

\author{
Andrea Gomes LINARDa, E milia Soares CH AVE Sa, \\ I saura L etícia T avares Palmeira RO LI M ${ }^{b}$, M aria I sis F reire de A G U IAR ${ }^{c}$
}

\section{RESUM 0}

O estudo objetivou analisar a compreensão dos enfermeiros a respeito dos princípios do Sistema Ú nico de Saúde universalidade, equidade e integralidade. Pesquisa descritiva realizada nos meses de agosto e setembro de 2008, por meio de entrevista semi-estruturada com 26 enfer meiras lotadas nas unidades básicas de Saúde de Fortal eza, Ceará. $\mathrm{N}$ a organização dos dados foi utilizada a técnica de análise de conteúdo. Os resultados apontaram que as enfer meiras concebem a univer sal idade como acesso universal de todos os usuários aos serviços de saúde, a equidade é 0 atendimento de forma igual itária a população, garantindo mais atenção aos menos favorecidos e a integralidade da assistência é a prestação de serviços nos três níveis de atendimento. Concluímos que os profissionais sinalizam possuir uma concepção dos princípios semel hante a que está presente na Constituição F ederal. Este fato representa um el emento importante para a concretização e for tal ecimento do model o de atenção à saúde vigente no Brasil.

D escritores: Equidade em saúde. Sistema Ú nico de Saúde. Serviços de saúde.

\section{RESUMEN}

E I estudio analizó la comprensión de los enfermer os con respecto a los principios del Sistema Ú nico de Salud universalidad, justicia eintegridad. E studio descriptivo realizado entre A gosto y Septiembre de 2008, a través de entrevistas semi estructuradas con 26 enfermeras de las unidades básicas de salud de F ortaleza, Ceará, B rasil. E n la organización de datos se utilizó la técnica de análisis de contenido. $L$ os resultados muestran que las enfer meras conciben la univer salidad como acceso universal de todos los usuarios a los servicios de salud, la equidad y la atención de forma igualitaria a la población, brindando más atención a los menos favorecidos e integralidad de la atención y la prestación de servicios en los tres niveles de atención. Concluimos que los profesionales señaliz an poseer una concepción de los principios semejante a la presente en la Constitución F ederal. E sto representa un el emento importante para concretizar y fortalecer el modelo de atención de salud vigente en Brasil.

Descriptores: E quidad en salud. Sistema Ú nico de Salud. Servicios de salud.

T ítulo: P rincipios del Sistema Ú nico de Salud: comprensión de los enfermeros de la E strategia de Salud de la Familia.

\section{ABST RACT}

The study aims to examine nurses' understanding of the principles of the $U$ nified $H$ ealth System: univer sality, equity and integrality. This is a descriptive study conducted from August to S eptember, 2008, through semi- structured inter views with 26 nurses of the basic units of $\mathrm{H}$ ealth from Fortaleza, Ceará, B razil. The technique of content analysis was used to or ganize the data. T he results show that nurses perceive universality as a universal access for all users of health services; equity as an equal attendance of the population, guaranteeing special attention to the poor; and integrality of care as the provision of services at all three levels of care. The conclusion shows that the practitioners' conception of these principles are similar to those presented in the F ederal Constitution. T his represents an important element for the implementation and strengthening of the model of health care in Brazil.

Descriptors: E quity in health. U nified $\mathrm{H}$ ealth System. $\mathrm{H}$ ealth services.

$T$ itle: Principles of the $U$ nified $H$ ealth System: nurses' conception of the Family $H$ ealth Strategy.

\footnotetext{
a D outora em E nfer magem, Professora A djunta do Curso de E nfer magem da U niversidade F ederal da I ntegração I nternacional da L usofonia Afro-Brasileira (U NILAB), Redenção, Ceará, Brasil.

${ }^{b}$ D outora em Enfermagem, Professora A djunta do Departamento de Enfermagem da U niversidade Federal do M aranhão (UFM A), São Luís, M aranhão, Brasil.

'E nfermeira, D outoranda pelo Programa de Pós-G raduação em E nfermagem da U niversidade F ederal do Ceará (U FC), Professora A ssistente do D epartamento de Enfermagem da U F M A, São Luís, M aranhão, Brasil.
} 


\section{INT RODUÇÃO}

O Sistema de Saúde no Brasil se originou influenciado por características trazidas desde o florescer do século passado, estas decorrentes de aspectos econômicos e políticos. Referido modelo de atenção a saúde foi alvo de influencias dos movimentos denominados Sanitarismo Campanhista, conferências nacionais de saúde e cartas de promoção da saúde.

0 nascimento da saúde pública no Brasil deuse na transição do século $X I X$ para o século $X X$ neste período o Sanitarismo Campanhista se configurou um processo de elaboração de normas e organizações sanitárias e de mudança nas práticas dominantes até então. Além deste movimento a VIII Conferência N acional de Saúde representou um marco histórico uma vez que houve o consenso da sociedade que para o setor da saúde no Brasil não era suficiente uma mera reforma administrativa e financeira, mas sim uma mudança em todo 0 arcabouço jurídico-institucional vigente, que contemplasse a ampliação do conceito de saúde ${ }^{(1)}$.

$N$ este efervescente cenário de discussão da saúde a proposta de reformulação do Sistema Nacional de Saúde parte de experiências que relataram ser o novo modelo assistencial viável, havendo ênfase para participação e colaboração da sociedade nas políticas públicas de saúde. A conjuntura social foi propícia para que a Constituição de 1988 incorporasse um conjunto de conceitos, princípios e diretivas extraídos das práticas correntes, havendo todavia uma reorganização desta prática na nova lógica referida pela reforma sanitária. A saúde na Constituição é definida como resultante de políticas sociais e econômicas, como direito de cidadania e dever do Estado, como parte da seguridade social e cujas ações e serviços devem ser providos por um Sistema Ú nico de Saúde ${ }^{(1)}$.

Com a criação do SU S e sua consequente regulamentação pelas L eis 8.080 , de 19 de setembro de 1990, e 8.142, de 28 de dezembro de 1990, ficaram claros conceitualmente os seguintes princípios: univer salidade de acesso aos serviços de saúde em todos os níveis de assistência; a integralidade de assistência, entendida como conjunto articulado e contínuo das ações e serviços preventivos e curativos, individuais e coletivos, exigidos para cada caso em todos os níveis de complexidade do sistema e a igualdade da assistência à saúde, sem preconceitos ou privilégios de qualquer espécie ${ }^{(1)}$.
A partir de então as outras Conferências Nacionais de Saúde direcionaram toda a sua atenção na implantação e consolidação do Sistema Ú nico de Saúde (SU S) que é constituído pel o conjunto de ações e serviços de saúde, prestados por órgãos e instituições públicas federais, estaduais e municipais, da administração direta e indireta e das fundações mantidas pelo poder publico.

A criação do SUS representou um avanço especialmente pelos seus princípios de organização que se traduzem em garantia de acesso de toda a população aos serviços de saúde e participação dos cidadãos no processo de formulação de políticas de saúde e controle da execução das mesmas. As ações e os serviços de saúde ofertados pela própria rede e os convênios que integram o SU S obedecem aos princípios: universalidade do acesso, integralidade e igual dade da assistência.

A pós 0 advento SU S, algumas estratégias foram formuladas no sentido de colocá-lo em prática. Como exemplos, podemos citar a criação dos distritos sanitários, dos sistemas locais de saúde e do Programa de Agentes Comunitários de Saúde (PACS). E sta última foi uma das principais, senão a mais importante das estratégias criadas em relação à assistência básica à saúde ${ }^{(2)}$.

$\mathrm{Na}$ continuidade desse processo, o M inistério da Saúde (M S) lança, no início de 1994 o Programa de Saúde da Família (PSF), posteriormente intitulado Estratégia de Saúde da Família (ESF), valorizando os princípios de territorialização, de vinculação com a população, de garantia de integralidade na atenção, de ênfase na promoção da saúde com fortalecimento das ações intersetoriais, de estímulo à participação da comunidade, e de trabalho em equipe com enfoque multidisciplinar, entre outros. O PSF surge com o objetivo de reorganizar a atenção básica do SU S, tendo 0 aspecto marcante de agilizar, de forma efetiva, a descentralização de serviços baseados nas reais necessidades da população, que se manifestam como prioridades e refletem problemas concretos ${ }^{(3)}$.

N esse processo histórico, a Atenção Básica foi gradualmente se fortalecendo, o que fez se constituir como porta de entrada preferencial ao Sistema Ú nico de Saúde, passando a ser o ponto de partida para a estruturação dos sistemas locais de saúde. N este cenário foi aprovada e publicada em 2006 a Política Nacional de Atenção Básica que traduz a marca da maturidade no que se refere a ESF na Atenção Básica em Saúde. Em seu bojo esta políti- 
ca orienta-se pelos princípios da universalidade, da acessibilidade e da coordenação do cuidado, do vínculo e continuidade, da integralidade, da responsabilização, da humanização, da equidade e da participação social(4).

A ESF veio reorganizar a prática da atenção à saúde e substituir o modelo tradicional, levando saúde às famílias, e, dessa forma almeja melhorar a qualidade de vida de todos que a buscam. 0 atendimento é prestado na unidade básica de saúde ou no domicílio pelos profissionais (médicos, enfermeiros, dentistas, auxiliares de enfermagem e agentes comunitários de saúde) que compõem as equipes da ESF.

$\mathrm{N}$ o leque de profissionais que integram a ESF a enfermeira desempenha relevante papel na execução de ações que permeiam as esferas gerenciais, clínica e educativas. No exercício de suas funções além da competência técnica, compromisso ético e responsabilidade se fazem necessário ao enfermeiro compreender e discutir de que forma a filosofia dos princípios doutrinários do SUS são concebidos.

Outros estudos corroboram essa colocação ressal tando a importância de se discutir a concepção da enfermeira sobre o processo da municipalização por entender que a enfermeira é um agente da equipe multidisciplinar em saúde estando diretamente envolvida com a consolidação do SU $S^{(5)}$.

A os longos dos 22 anos de existência do SUS diversas experiências vem buscado descrever, compreender experiências e avaliar o sistema de saúde considerando a participação efetiva dos sujeitos envolvidos no cotidiano dos serviços - usuários, gestores e profissionais de saúde, a fim de reorganizar as práticas assistenciais em saúde ${ }^{(6)}$. As complexas demandas do SU S e seu aparato operacional vinculado à garantia da integralidade da atenção, da humanização da assistência, da construção da interdisciplinaridade e do trabalho em equipe demandam intensos processos de reflexão e discussão(7).

A credita-se neste estudo que a percepção filosófica da enfermeira irá influenciar no processo de trabal ho da equipe redirecionando sensivel mente a práxis das ações de atendimento, bem como, no tocante a otimização dos recursos financeiros, humanos e materiais.

Assim, esse estudo justifica-se na medida em que a sua execução oportunizará mais elementos para a reflexão dos profissionais acerca da univer salidade, equidade e integ ralidade em saúde, possibilitando um novo olhar para a assistência prestada aos usuários que procuram atendimento nas U nidades Básicas de Saúde (U BS).

Diante dessa conjuntura se questiona: 0 enfermeiro que compõe a equipe do ESF compreende na íntegra o real significado dos princípios integralidade, universalidade e equidade no contexto de trabalho da U BS? De que maneira o enfermeiro concebe o cumprimento destes princípios na sua prática de atendimento?

Estes questionamentos emergem, pois partimos da premissa que a compreensão dos profissionais a respeito de saúde, assim como, do ordenamento jurídico que subsidia o direito a saúde apresentará desdobramento na qualidade do atendimento prestado a população.

Considerando esse aspecto e na tentativa de responder as indagações citadas elaboramos o seguinte o objetivo de analisar a compreensão dos enfermeiros a respeito dos princípios do SUS universalidade, equidade e integ ralidade.

\section{MÉTODOS}

Estudo descritivo com abordagem qual itativa. Compuseram a pesquisa 26 enfermeiras lotadas nas unidades básicas de saúde em Fortaleza vinculadas a ESF.

A coleta de dados aconteceu durante os meses de agosto e setembro de 2008 , onde foi utilizado um roteiro de entrevista com questões a respeito do conhecimento e cumprimentos dos princípios do SUS no atendimento. As entrevistas aconteceram na U nidade Básica de Saúde sempre após o expediente de atendimento das enfermeiras em uma sala reservada na unidade para essa atividade.

Os dados foram organizados a partir das transcrições das entrevistas e leituras sucessivas das falas, onde as idéias centrais, ou seja, aquelas mais evidentes, que descreveram de forma sintética e precisa o sentido das falas, foram identificadas e registradas. As transcrições foram organizadas pela técnica de Análise de Conteúdo(8). Ao final identificamos as categorias: Interpretação conceitual dos princípios oriunda da visão das enfermeiras sobre princípios do SU S, durante o serviço prestado em unidade básica de saúde e C umprimento dos princípios que apresenta a dinâ- 
mica empregada pela enfer meira para dar continuidade ao atendimento prestado ao usuário de forma que este usufrua de todos os níveis de atenção à saúde.

Com o intuito de obedecer às diretrizes que regulamentam os estudos que envolvem seres humanos, para as informantes desta pesquisa foi apresentando o T ermo de Consentimento Livre e E sclarecido, com dados referentes ao caráter voluntário do estudo e possibilidade de desistência a qualquer momento. Para garantir o anonimato, as fal as foram identificadas pelo código E nf. seguido do número de ordem. 0 estudo na fase de projeto de pesquisa foi enviado para o comitê de ética da U niversidade de Fortal eza e aprovado segundo o parecer 216/ 08 .

\section{RESULT ADOS E DISCUSSÃO}

A maioria das enfermeiras graduou-se há 10 anos e, portanto tem proximidade conceitual com o ordenamento jurídico do SU S. I nseridas na ESF estão há 08 anos. $\mathrm{Na}$ formação lato sensu todas as profissionais possuem especialização na área de saúde pública. Esse tipo de especialização apresenta em seus conteúdos temais tais como: políticas de saúde do Brasil com ênfase ao Sistema Ú nico de Saúde.

A seguir descrevemos as falas das enfermeiras que direcionaram a construção da categoria Interpretação conceitual dos princípios. As falas são apresentadas e ag rupadas de acordo com 0 princípio que remetem em seguida apresentamos a discussão.

U niversalidade é 0 acesso universal [ igualitário] a todos os usuários do SU S (Enf. 01, 29 anos).

U niversalidade nos devemos atender a todos os pacientes independentes da sua idade, sua condição sócio econômica, condição educacional. E ntão o conceito da U niversalidadeé 0 atendimento a todos, todos queprocuram o serviço (Enf. 02, 34 anos).

U niversalidade todos tem direito, acesso aos serviços de saúde (E nf. 03, 36 anos).

U niversalidadenão ter distinção de cor, raça. Participação popular é o povo interagir (E nf. 04, 34 anos).

Com relação à U niversalidade, é você ter condição de atender as pessoas, todas as pessoas de qual quer lugar, certo? (Enf. 05, 29 anos).
U niversalidade é a questão de direito a saúde que todos têm. E ntão assim, é 0 acesso a saúde, onde todo 0 cidadão deve ter 0 acesso garantido à saúde de uma forma universal (Enf. 06, 42 anos.).

A compreensão expressa pelos profissionais do termo univer salidade como 0 acesso aos serviços de saúde sem discriminação de qualquer ordem vem a complementar a afirmação de autores que compreendem universalidade na concepção de garantia da cobertura dos riscos sociais de sua população em um E stado democrático de direitos ${ }^{(9)}$.

$\mathrm{Na}$ cobertura dos riscos sociais a desigualdade da oferta dos serviços de saúde se constitui um entrave para a plena univer salização. E ssa desigualdade é fruto da construção espacial do sistema de saúde em ritmos diferenciados nas regiões do país. Superar essas desigualdades tem requerido dos profissionais de saúde além do conhecimento filosófico que norteia a organização do sistema algumas condições estruturais mínimas para a continuidade do processo de implantação da universalidade da saúde.

De posse das condições estruturais mínimas associado à presença dos agentes comunitários de saúde (ACS) será possível trabal har vislumbrando estratégias concretas de redução das desigualdades na oferta dos serviços. A presença do ACS no território brasileiro tem contribuído para a redução de alguns indicadores de morbi-mortalidade, além da mel hora referente à prestação de serviços ${ }^{(1)}$.

As falas a seguir retratam a compreensão dos profissionais enfermeiros a respeito do princípio integralidade de assistência.

Integralidade éver o indivíduo como um todo, garantindo atendimento na atenção primaria, secundaria e terciário (E nf. 07, 29 anos).

A Integralidade que essa pessoa, esse sujeito, deve ser atendido em todos os níveis de atenção. N ível primário, secundário e terciário (E nf. 08, 34 anos).

Integralidade: o SUS da direito a pessoa satisfazer suas necessidades em todos os níveis de atenção. Começando na atenção básica [ hierarquização] (Enf. 09, 36 anos).

Integralidade é atender no todo o cliente [ resolutividade] (Enf. 10, 34 anos).

A Integralidade é você conseguir dar continuidade ao tratamento da atenção primaria, secundaria e terciária (Enf. 11, 29 anos). 
A Integralidade eu vejo como forma de se trabalhar a saúde, numa forma bem integrada nos seus diversos setores, nos seus diversos níveis primário, secundário e terciário (E nf. 12, 42 anos.).

As profissionais compreendem integralidade relacionada à idéia de atendimento em todos os níveis de atenção com resolução de problemas. A integralidade se desdobra na adoção dos meios necessários para a efetivação do cuidado, como: consulta médica, consulta de enfermagem, exames, internação, atividades educativas, tratamento, entre outros. I mplica dispor tipos diferentes desses meios segundo o grau de complexidade da atenção à saúde, ou seja, exames para a atenção básica, para a média e alta complexidade; da mesma forma os medicamentos: uso ambulatorial e na atenção hospitalar.

A garantia do princípio da integralidade refere-se à garantia de acesso do cidadão aos diversos níveis de atenção e complexidade buscando promover, prevenir, restaurar a saúde e reabilitar os indivíduos(10).

No contexto de atenção integral a todos os usuários de um sistema público de saúde o Brasil ainda não apresenta condições para atender integralmente às necessidades de atenção a saúde de todas as pessoas, nesta conjuntura mesmo dispondo de um substancial aumento dos recursos investidos no setor saúde e nos determinantes sociais responsáveis pela maioria das más condições de saúde não reverteríamos o quadro porque a população cresceu nas últimas décadas sensivel mente e na contramão desse crescimento o desenvolvimento das cidades acontece em ritmo lento.

0 entendimento do ter mo equidade na ótica das entrevistadas revela os seguintes aspectos:

0 princípio da E quidade devemos atender a todos de forma igual itária respeitando as limitações e carências de cada individuo, fazendo com isso, que eu atenda ele de forma mais especial, aquele que mais necessita. E ntão a equidade nesse sentido seria dar mais a quem pre cisa mais (Enf. 13, 34 anos).

E quidade é 0 atendimento de forma igualitária a todos os usuários, garantindo mais atenção aos menos favorecidos (Enf. 14, 29 anos).

E quidade é estabel ecer prioridades de necessidade no atendimento. Atender de maneira desigual os desiguais (Enf. 15, 36 anos).
E quidade é dar mais a quem tem menos (E nf. 16, 34 anos).

E quidade é você atender todo mundo de forma igual dando mais a quem tem menos, e dando menos a quem tem mais (Enf. 17, 29 anos).

A equidade é aquela historia dar mais a quem tem menos, agente ta trabal hando de uma forma mais igualitária, para o individuo (E nf. 18, 42 anos).

A compreensão referida pelos profissionais esta associada à igualdade de atendimento buscando garantir mais atenção à saúde das pessoas que mais necessitam. D essa forma mais do que tratar todos iguais, a equidade albergaria uma dependência ao valor de justiça, ou seja, de se buscar dar mais a quem precisa mais.

N este sentido, é preciso ter clara a noção de direito de igualdade antes de discutir equidade, porque também eqüidade não significa retirar direitos, mas respeitar o direito de que todos são iguais e buscar dar mais prioridade àquele que mais precisa. Com esta compreensão, a eqüidade em saúde implica em prover a cada um a atenção, as ações de saúde segundo suas necessidades(11).

Equidade pressupõe a garantia de acesso de qualquer pessoa em igualdade de condições aos diferentes níveis de complexidade do sistema, de acordo com a necessidade de cada situação fica assegurado que as ações coletivas desenvolvidas pelo Estado em parceria com os profissionais de saúde serão dirigidas por prioridades ampla e publicamente reconhecidas. A lcançar este estágio de desenvolvimento se configura um dos grandes desafios do Brasil.

$\mathrm{N}$ a realidade, a oper acionalização do conceito de equidade exige tal grau de sofisticação e de delicadeza da política, da gestão e da própria organização social que em poucas situações concretas foi possível observar-se sua aplicação efetiva ${ }^{(12)}$.

Conhecer e compreender os princípios do SU S, bem como o conceito ampliado de saúde pode ser indispensável para produzir mudanças na saúde segundo sua realidade local. Todavia, apenas o conhecimento e apreensão adequada dos princípios e diretrizes constitucionais por parte das enfermeiras no processo de construção do SU S, não significa necessariamente que ocorrerá uma efetivação do arcabouço doutrinário do sistema na atenção básica. Esta política de saúde se configura um processo em per manente construção que al- 
meja, a médio e longo prazo uma mudança no paradigma de atenção à saúde e a busca de um sistema de saúde eficaz, eficiente, de qual idade e eqüitativo. A dialética da inter pretação dos termos pelos enfer meiros revela a busca pela compreensão do contexto filosófico do atual modelo de saúde e o reconhecimento de sua importância no cenário de trabalho.

O cumprimento dos princípios do SU S implica um passo a mais, cujo conhecimento das necessidades e demandas individuais e coletivas acerca da saúde deve ser resultante da permanente interação dos atores na relação demanda e oferta, nos diversos níveis de atenção à saúde individual e coletiva. Violar um princípio é muito mais grave que transgredir a uma norma qualquer. A desatenção ao princípio implica ofensa não apenas a um específico mandamento obrigatório, mas a todo um sistema de comandos ${ }^{(13)}$. Diante desta conjuntura, elaboramos a categoria Cumprimento dos princípios a partir das seguintes falas:

B om a gente tenta, é o profissional de saúde principalmente na área pública, a gente tenta é todos os dias estar exercendo as nossas atividades dentro dos princípios do SU S, é difícil sim, édifícil porque a gente vive diante de uma realidade que nos temos muitas dificuldades (Enf. 19, 42 anos).

$\mathrm{H}$ oje em dia o acesso à saúde eu acredito que ta bem melhor, o indivíduo vem ate o posto de saúde, o PSF é um programa que nos temos esse el o com a comunidade, de trazer a comunidade ate o serviço de saúde, eles tem esse acesso, porém nos temos um viés que dificulta um pouco, a questão ainda da oferta de serviço, que não esta sendo dada da forma que épra ser dado (E nf. 20, 40 anos).

E mbora existam diversas dificuldades para a oferta de serviços em quantidade adequada existe por parte dos profissionais o desejo em superar os obstáculos para o cumprimento de ações direcionadas ao atendimento da atenção primaria. 0 conhecimento e busca do cumprimento dos princípios éo alicerce para o alcance de uma melhor qualidade das ações e serviços voltados para a promoção da saúde, prevenção, recuperação e reabilitação.

Para cumprir a lei todos os municípios deveriam implantar o SUS nas suas áreas de influência de acordo com os princípios de diretrizes. Se a realidade que observamos ainda é diferente significa que novos esforços são necessários para que os dirigentes e técnicos cumpram e façam cumprir a lei ${ }^{(14)}$.

Observamos ainda que as enfermeiras reconhecem a importância da sua participação para a concretização do atual modelo de saúde, apesar dos limites impostos à sua atuação, o que conduz a uma per spectiva de acreditar que a construção deste sistema vai se consolidando conforme sua conjuntura administrativa e política. N este sentido o profissional poderá ser influenciado, por estar inserido nessa conjuntura como é percebido nas falas a seguir:

E $u$ acr edito que sim, a gente tem muitas atividades como enfermeira generalista aqui na estratégia da saúde da família, isso faz com quea gente cumpra esses princípios mesmo queagente não queira e mesmo quea gente não conheça basta ter bom senso e ter um coração que entenda o que é a necessi dade de saúde de uma pessoa (Enf. 21, 38 anos).

M esmo que a pessoa não saiba o que é universalidade ela tem que saber que a pessoa que chegou à unidade tem uma necessidade e el a como enfermeira ou médico, tem que saber que a pessoa tem a necessidade e tem que fazer com que isso se realize (E nf. 22, 38 anos).

A enf. 21 revela que por atuar como generalistas contribui para o cumprimento dos princípios, uma vez que, a mesma não trabalha na perspectiva reducionista do atendimento a queixa, mas sim na leitura de compreender a dimensão da necessidade de saúde e solucioná-la.

A prática de enfermagem, nesse contexto, é concebida como um trabalho coletivo desenvolvido por profissionais de enfermagem e articulado às práticas dos demais trabalhadores da área de saúde. Essa prática visa a identificação das demandas de assistência a saúde do homem, bem como seus fatores determinantes ${ }^{(15)}$.

\section{CONSIDERAÇÕES FINAIS}

N este estudo nos propomos a revisitar os princípios do SU S na ótica das profissionais enfermeiras vinculadas a Estratégia Saúde da Família (ESF). Verificamos que as profissionais se revestem de conhecimento a respeito das concepções e doutrinas que permeiam o Sistema Ú nico de Saúde. Os princípios universidade, integralidade e equidade são conceituados de forma correta havendo o reconhecimento da importância de seu cumprimento na 
prática clínica para superar al guns desafios inerentes a oferta dos serviç̧os e ao acesso da população a estes.

0 desenho constitucional de 1988 que nos remeteu ao SU S é correto e moderno, mas que carece de condições e tempo para afirmar-se socialmente. $E$ entender que seu desenvolvimento conseqüente vai depender da formulação estratégica de uma agenda para a saúde a ser perseguida, tenazmente, com muito esforço e por muitos anos.

$\mathrm{Na}$ busca por responder as complexas demandas do SU S, a consolidação da ESF e seu aparato operacional poderão os gestores e profissionais de saúde estar criando espaços para aprofundar as discussões sobre as concepções saúde, bem como, sobre as políticas públicas existentes, trabalhando pela construção de práticas em saúde baseadas na compreensão dos princípios e inserindo 0 acolhimento, a humanização e o cuidado no seu cotidiano de trabalho.

\section{REFERÊNCIAS}

1 Andrade LOM, Pontes RJS, M artins Junior TA Descentralização no marco da Reforma Sanitária no Brasil. Rev Panam Salud Publica. 2000;8(1/ 2):85-91.

2 Espínola FDS, Costa ICC. A gentes comunitários de saúde do PACS e PSF : uma análise de sua vivência profissional. Rev Odontol U niv Cid São Paulo. 2006; 18(1):43-51.

3 Souza M FA. Enfermagem reconstruindo sua prática: mais que uma conquista no PSF. R ev Bras E nferm. 2000;53(n esp):81-6.

4 M inistério da Saúde (BR), Secretaria de Atenção à Saúde, D epartamento de A tenção Básica. Política nacional de atenção básica. Brasília (D F ); 2006.

5 Borges M ASF, Nascimento MAA. A concepção da enfermeira sobre o SU S: um caminho sem volta. Rev Bras Enferm. 2005;58(3):272-7.

\section{Endereço da autora / Dirección del autor / Author's address:}

Andrea $\mathrm{G}$ omes Linard

Rua Benjamim M oura, 489, ap. 201-A,

Cidade dos $\mathrm{F}$ uncionários

60822-480, Fortaleza, CE

E-mail:linard@unilab.edu.br
6 Ferreira VA, Acioli S, Heringer A, Barros ALS. Os princípios do Sistema Ú nico de Saúde nas práticas educativas dos enfer meiros no P rograma de Saúde da Família. Online Braz J N urs [ I nternet] . 2006 [ citado 2010 set 06];5(3). Disponível em: http:/ / www. objnursing.uff.br/ index.php/ nursing/ article/ view/ 469.

7 Costa R KS, M iranda FAN . Sistema Ú nico de Saúde e da família na for mação acadêmica do enfer meiro. Rev Bras Enferm. 2009;62(2):300-4.

8 Bardin L. Análise de conteúdo. Lisboa: E dições 70; 2004.

9 M arques RM, M endes A. Democracia, saúde pública e univer sal idade: 0 difícil caminhar. Saúde Soc. 2007; 16(3):35-51.

10 Campos CEA. 0 desafio da Integ ralidade segundo perspectivas da vigilância da saúde e da saúde da família. Ciênc Saúde Colet. 2003;8(2):569-84.

11 Souza RR. Políticas e práticas de saúde e equidade Rev E sc Enferm USP. 2007;41(n esp):765-70.

12 Sousa CGW. Reflexões temáticas sobre equidade e saúde: 0 caso do SU S. Saúde Soc. 2006;15(2):2333.

13 Salazar AL, Rodrigues K, N unes Júnior VSN. A assistência privada à saúde: regulamentação, posição IDEC e reflexos no sistema público. In: M inistério da Saúde (BR ). Direito sanitário e saúde pública. Brasília (DF); 2003. v. 1; p. 333-62.

14 Paim JS. 0 que é o SU S. Rio de Janeiro: Fiocruz; 2009.

15 Santos BRL, Paskulin LG, Rosa LG, W itt RR, D ias JS. $O$ trabal ho em saúde coletiva e a enfermagem: concepções dos gerentes das unidades de saúde de Porto Alegre. Rev G aúcha Enferm. 1999;20(n esp): 102-12.

Recebido em: 05/ 03/ 2010

A provado em: 08/ 02/ 2011 